\title{
Notas para una propuesta lógico-conceptual de la relación entre las interacciones y cooperación en redes de política pública
}

\author{
Cristo Avimael VÁzQuez Ceballos \\ Universidad Autónoma de Estado de México \\ c_avimael@yahoo.com.mx
}

Recibido: $10 / 04 / 2015$

Aceptado: 4/06/2015

\begin{abstract}
Resumen
En muchos sectores de política pública, paulatinamente, se han desarrollado flujos de gobierno más interactivos, etiquetados como gobernanza y materializados a través de una serie de redes de política pública en las cuales interactúan actores gubernamentales, sociales, civiles, ciudadanos, agentes del mercado, entre otros, a modo de cooperar en torno a objetivos comunes. Ante tal contexto, este trabajo argumenta que la variabilidad de sus interacciones y que unas son más conducentes, que otras, para la cooperación debido a ciertos atributos estructurales de la red (heterogeneidad e integración). Las conclusiones sugieren que según varíen dichos atributos se propiciarán interacciones asociativas, a nivel de acciones combinadas o superficiales, siendo las asociativas las más propicias para la cooperación.
\end{abstract}

Palabras clave: gobernanza, políticas públicas, propiedades de la red, mecanismos de vinculación y patrones de interacción.

\section{Notes for a logical and conceptual proposal about relationship among network interactions and cooperation in policy networks}

\begin{abstract}
In several policy areas, we have seen the gradual development of more interactive forms of governing, usually labeled as governance, which have took the form of policy networks. In policy networks, governmental actors interact with social, civil and market players, among other relevant stakeholders, seeking their cooperation. In this context, this paper argues that exists a relationship between of the variability of policy networks and cooperation among actors, having in mind that the structural attributes (heterogeneity and integration) mediate this relationship. The article concludes that the variability of these attributes will foster combined actions, superficial interactions and partnerships, being this last kind the more propitious to foster cooperation.
\end{abstract}

Key words: gobernance, policy, network properties, resources, linking mechanism and interacting patterns.

\section{Exordio}

Este trabajo gira en torno a las crecientes interacciones del gobierno con actores público-sociales y agentes del mercado para atender sectores de política y cuyas expresio- 
nes cobran la forma de redes de política pública (RPP). En términos de Aguilar (2014), en los tiempos de la gobernanza, a través de este tipo de estructuras, el gobierno, agentes del mercado o privado-lucrativos y actores público-sociales (sean ciudadanos a título personal o a través de sus organizaciones, ambos distinguiéndose por cuanto desde lo social trabajan en torno a objetivos colectivos, ya sea independientemente o en relación con el gobierno) interactúan a efecto de definir, acordar y decidir sus principios y objetivos comunes, los recursos y las actividades que se consideran son necesarias y conducentes para realizar y concretizar sus intereses generales. En consecuencia, el problema es que (como en todo proceso de acción organizada) tales interacciones pueden redundar en el despliegue de procesos cooperativos o no cooperativos, mermando así la capacidad de incidencia de las redes en las políticas públicas a efecto de resolver o atender problemáticas sociales.

En tal tesitura, más allá de las connotaciones descriptivas y conceptuales que han dominado las aportaciones en torno a la gobernanza, es preciso considerar que el proceso de gobernanza no es aleatorio, discrecional o fortuito, sino que genera estructuras interactivas o redes de política pública que hacen posible o no «que los actores públicos, privados y sociales conozcan las dimensiones y las causas de los problemas que quieren atender o las metas que quieren alcanzar, asignen y empleen de manera eficiente los recursos que son necesarios para resolver los problemas y alcanzar las metas de su interés» (Aguilar, 2014: 17).

Así las cosas, a modo de reconocer factores de importancia a tener en cuenta al estudiar problemas como el señalado, el objetivo de este trabajo es proponer una relación lógico-conceptual entre las interacciones de los integrantes de una red de política pública y los procesos cooperativos que propicia la red al atender problemáticas colectivas. En tal tesitura, la presente colaboración ubica su aparato crítico en las inferencias que ha realizado el análisis de redes sociales; por lo cual, la pregunta que sirve como guía metodológica nos conduce a indagar ¿por qué ciertas interacciones, léase patrones de interacción, son más conducentes para la cooperación que otras?

En razón del objetivo planteado, se retoma la propuesta de Crozier y Friedberg (1980) dado que facilita, de entrada, comprender que la estructuración de la red impone oportunidades y desventajas a la acción de los actores con miras a favorecer objetivos de cierto modo compartidos. Esta contribución angular, se complementa con el Análisis de Redes Sociales (ARS) (Knoke y Yang, 2008 y Wasserman y Faust, 1994) a efecto de reconocer que hay cualidades estructurales de las redes de política pública que importan a la hora de entender los resultados de la cooperación y a la cooperación misma. Y para especificar estas cualidades estructurales, recurro a las aportaciones realizadas en torno al Capital Social (CS), en las cuales se ponderan dos atributos estructurales (integración y heterogeneidad) como factores que inciden en la capacidad de los actores para vincularse en acciones colectivas (Burt, 2000).

Como antecedentes a los planteamientos expuestos, encontramos que las temáticas han variado sobre temas relacionados a la gestión de redes (Kickert, Klijn y Kooppenjan, 1997), las redes de políticas como formas específicas de gobernanza y de intermediación de intereses (Mayntz, 2000 y Rhodes, 1997) y las redes de gobernanza local en temas como política urbana, educativa, salud, drogas, etc. (Stoker, 1998). En un contexto hispano, encontramos que el debate se ha orientado a revisar y estructurar la 
literatura norteamericana y europea en torno al tema. Así mismo, estos trabajos redundan en la utilidad de las redes de políticas como nuevas formas de entender las relaciones Estado-sociedad-mercado, ya sea como metáforas para describir las intermediaciones de intereses o como modelo analíticos de gobernanza (Luna y Velasco, 2009; Sánchez, 2008; Guarneros, 2007; Natera, 2005; Zurbriggen, 2003; del Castillo, 2000; Poggiese, 2000; y Bravo, 1997). Trabajos de orden más empírico, se han propuesto contrastar las posibilidades y limitaciones del enfoque de redes de políticas en las realidades latinoamericanas como Nigenda y González (2009), Vargas (2008), Porras (2007), Mariñez (2007), Guarneros (2007), Porras (2001) y Poggiese, Rendín y Alí (1999). Así como hay otros estudios que disciernen acerca de las implicaciones normativas para el espacio público y ejercicio de la ciudadanía de las redes de políticas según los estudios de Zabaleta (2009) y Luna (2005). En tanto que hay otra tendencia con pretensiones de generar estrategias aplicables a la gestión de redes de políticas, casos ilustrativos son Zabaleta (2008) y Aragón (2001).

De los antecedentes citados cabe destacar que la utilidad principal del vocablo de red de política pública ha sido descriptiva y comprensiva, lo cual indica la subutilización del potencial explicativo de las redes de políticas públicas. Ante tal estado de cosas, se advierte que no importa únicamente reconocer las construcciones societales que se vienen gestando en el marco de las transformaciones del Estado y que el estudio de tal fenómeno en particular se ancle en cuestiones descriptivas. De hecho, el quehacer académico en redes de políticas poco ha observado a su objeto de estudio como una unidad conceptual con potencial explicativo, y así lo dejan observar aportaciones de Berardo (2009), Percival (2009), Sandström (2008), Lewis (2005), Howlett (2002) y Provan y Milward (1995), mismas que ubican a las redes de políticas públicas en el campo de las variables. De ahí, la pertinencia de este trabajo por establecer un marco lógico-conceptual que coadyuve, en el contexto de las aportaciones precedentes, a identificar elementos constitutivos de la dimensión estructural-interactiva de las redes de políticas públicas como factor explicativo de los procesos cooperativos o no cooperativas que propicia.

En términos de la presente colaboración entiendo a las redes de política pública como «patrones de interacción más o menos estables entre actores interdependientes y articulados en torno a problemas y/o programas de políticas públicas» (Kicker, Klijn y Koppenjan, 1997: 6). Desglosando esta definición, una red de política pública implica: (i) interdependencia entre actores -sean individuos que participan a título personal u organizaciones -, (ii) interacción entre los integrantes de la red dado el intercambio de recursos y la negociación de sus intereses, en el marco del problema o asunto público en mano, y (iii) un modo de organización heterárquico.

La organización de este documento se esquematiza en cuatro apartados generales y las conclusiones. En un primer momento, se discute la naturaleza intencional y deliberada de una red de política pública al ser conformada por individuos autónomos y racionales, lo cual conduce a explorar las relaciones de poder entre ellos a partir de sus recursos (materiales y simbólicos) que portan o controlan a modo de ganar ventaja en la priorización de decisiones a implementar. Esto hace pensar a las redes como constructos que tratan de articular múltiples racionalidades (e intereses) instituyendo un mínimo de organización para propiciar procesos cooperativos. 
En un segundo apartado, se piensa a las redes de política pública a partir de dos atributos estructurales (integración y heterogeneidad) y que cobran importancia a la luz de los preceptos teóricos sobre el capital social. En un tercero, se reconoce que la relación entre las decisiones de los integrantes de las redes y su estructura, ocurre mediante una serie de interacciones que, lejos de ser estables y uniformes, se caracterizan más por su carácter variable debido a la integración y heterogeneidad de la red de política pública.

Ya en un cuarto apartado, a partir de distinguir los mecanismos de vinculación entre los actores de una red de política pública y las funciones organizativas claves en este tipo de redes, es que se proponen tres patrones de interacción (asociación, acciones combinadas y superficiales) y se establece que según sea el nivel de integración y heterogeneidad, se propiciará una u otra forma de interacción. Para cerrar este documento, presento una recapitulación breve y esbozo las implicaciones de esta propuesta para el estudio de las redes de política pública, sugiriéndose suponer a las interacciones como mediación entre los comportamientos de los actores que se integran en redes de política pública y sus posibilidades de cooperación. Más específicamente, dichas interacciones se caracterizan por ser variables, y según se comporten dos cualidades estructurales (heterogeneidad e integración), se propiciarán interacciones a nivel de asociación, acciones combinadas y superficiales.

\section{Supuesto de partida: las redes de política pública como estructuras para propiciar la cooperación}

El estudio del comportamiento organizacional y de las decisiones (públicas) desde el último tercio del Siglo XX estuvo dominado por la teoría de la decisión racional que otorga un lugar preponderante a la elección individual. Este advenimiento resultó ser una respuesta de los teóricos de la economía política al funcionalismo y al marxismoestructuralismo que coincidían en señalar las determinaciones objetivas sobre la acción del individuo con capacidad de voluntad, estrategia, decisión y persecución de objetivos propios (Vergara, 1997), es decir, de actuar deliberadamente, según supone la percepción racional del comportamiento humano.

En el enfoque de la decisión racional clásica, el entendimiento más común es que durante un proceso de decisión el individuo posee información completa sobre las alternativas y sus preferencias respecto a ellas son estables (Vergara, 1997). Desde esta perspectiva se asume que el proceso de decisiones públicas, en el cual el individuo es el gobierno, se desarrolla en fases bien establecidas (formulación, decisión e implementación) basado sobre la afirmación de que el decisor gubernamental primero analiza el problema y las alternativas de solución, y luego confecciona unilateralmente una decisión considerando la opción elegida (Modelo I en Allison,1969). Este enfoque de decisión sería cuestionado por teóricos como Lindblom (1979) y Simon (1976), considerando que la información con la cual se hacen decisiones nunca es perfecta y que los individuos están imposibilitados (limitados) para examinar todas las alternativas posibles, a pesar de que las preferencias sean estables y conocidas por ellos. Esta segunda perspectiva se basa en la idea de que los tomadores de decisiones no tratan de optimi- 
zar su beneficio al tomar una decisión sino sólo de encontrar un curso de acción que les proporcione resultados aceptables dado los costos para adquirir información y las limitaciones del decisor para procesarla, desvirtuándose así al gobierno como un agente racional y omnipotente.

No obstante las divergencias entre ambos enfoques, los dos convergen en que los procesos de decisión pueden ser estudiados considerando a un único actor. Esto en línea con el individualismo metodológico. Por ello, más adelante se discutiría que los procesos de decisión pública involucraban una multiplicidad de actores y que las políticas eran consecuencia de procesos interactivos más que de la decisión unilateral de un único actor (Bardach, 1978 y Pressman y Wildavsky, 1973) y que en todo caso el gobierno más que ser un actor unificado es una multiplicidad de actores interconectados débilmente por numerosas reglas (Olsen, 1992 y March y Olsen, 1989), se reconocería la influencia del contexto institucional en el cual se decidía (Berman, 1978), se distinguiría la naturaleza política inevitable de todo proceso de política pública (Allison, 1969), además de que ya se consideraba que los agentes operan con recursos limitados a la hora de tomar una decisión (March y Simon, 1958).

Por lo que la de decisión o política pública más que ser un conjunto de fases perfectamente establecidas es un proceso complejo debido al número de actores que toman parte o tratan de influir en ella, las preferencias de los involucrados no siempre están perfectamente bien establecidas por las percepciones sobre lo público, las estrategias y soluciones al problema cambiantes en el tiempo. Entonces, tomando en cuenta el enfoque racional (sea el clásico o el limitado) que asigna al individuo ciertas preferencias y capacidades de discernir sobre las diferentes alternativas en orden de escoger la acción más favorable y con capacidad de deliberar acerca de los medios más óptimos para sus fines, así como considerando la complejidad del proceso de las decisiones públicas es que se piensa que las políticas públicas implican a actores racionales que interactúan en sus procesos. Ello traído a la idea de redes implica asemejarlas como agregados de individuos que deciden involucrarse en procesos complejos de política pública dada su voluntad, estrategia y decisión a fin de concretizar objetivos (propios y probablemente compartidos) sin que exista la certeza de que la red conformada trabaje para favorecerlos. Es decir, queda el supuesto de que las redes de política pública son establecidas por las acciones deliberadas de actores con cierta racionalidad e intencionalidad.

Si consideramos que las redes se hacen y deshacen, y más aún que las mismas se construyen con miras a lograr la cooperación, esto indica que la activación y desenvolvimiento de las redes son expresión de acciones intencionadas y tomadas por actores con intereses propios y distintos pero a fin de cuentas con cierto grado de interdependencia.

Así es como la existencia de objetivos en común no es un condicionamiento necesario para la integración de diversos actores y su articulación en redes (Sandström, 2008), como tampoco que dicho fenómeno sea natural y derivado del desarrollo de las interacciones y consecuencia lógica de los problemas a resolver (Crozier y Friedberg, 1980). Más bien, los actores se vinculan voluntariamente a fin de adquirir recursos (de cierta manera escasos) que se encuentran dispersos entre ellos con miras a satisfacer su umbral de intereses y objetivos específicos. Decir que un actor (organizacional o indi- 
vidual) «tiene intereses en una política significa que el proceso y los resultados de una decisión tiene consecuencias para éste» (Knoke et al., 1996: 12). Y muchas de las veces, el escenario de oportunidad para acceder a los recursos e influir en la construcción de las acciones públicas lo representa el tratamiento de los problemas públicos que implican la programación de múltiples objetivos colectivos. Por eso, entre gobierno y sociedad, pero también entre diversos actores sociales, entre el sector gubernamental y privado-lucrativo, han emergido muchas formas de interdependencia, siendo una de ellas las RPP.

En tal tesitura, las redes de política pública se desarrollan e integran porque los actores se necesitan unos a otros. De hecho, la interdependencia es una precondición de las redes (Klijn, 1997) que se da cuando uno no controla todos los recursos necesitados para una acción deseada (Pfeffer y Salancik, 1978) pero que se desea acceder a ellos. Así, es necesario reconocer las interacciones entre los actores a fin de generar intercambio de recursos tanto materiales como simbólicos que si bien acciones voluntarias no necesariamente exentas de problemas dado su carácter interactivo, colectivo y político.

Por lo tanto, las redes de política pública como un reflejo de la acción voluntaria de los actores que las integran y de las relaciones de interdependencia entre ellos, estructuran una solución alternativa para propiciar la cooperación o acción conjunta de múltiples actores.

\subsection{Las redes de política pública como alternativa para la cooperación}

Hablar de la cooperación, es decir de la acción organizada de los hombres o conducta de grupo con miras a cumplir objetivos comunes (Crozier y Friedberg, 1980), implica reconocer los posibles medios para cooperar. Antes de las contribuciones de Hardin (1968) y Olson (1965), básicamente se asumía que los individuos, una vez identificados los objetivos comunes, procedían a organizarse y cooperar (actuar colectivamente) para llevarlos a cabo. A partir de esos trabajos seminales, comenzó a prestarse atención a algunos problemas que dificultaban la cooperación.

A estas primeras contribuciones teóricas, en la década de 1980 se abriría la discusión sobre cómo llevar a cabo la cooperación o las soluciones a los problemas de la acción colectiva. La propuesta de Ostrom (1990) se ha perfilado en esta tendencia, al indicar que hay contextos o factores institucionales que facilitan que los actores voluntariamente cooperen mientras otros contextos son menos conducentes para ello, siendo necesarios ciertos mecanismos coercitivos que incentiven a cooperar, indicando dos: el Estado y el mercado. A este respecto, Klijn (1997) argumenta que a esos mecanismos extremos se pueden considerar las redes de política pública. Es decir, se perfila el supuesto de las redes de política pública como alternativa para propiciar la cooperación entre agentes racionales y muy probablemente distintos entre sí.

Las RPP, como una opción para generar procesos cooperativos, cobra relevancia ya que en orden de lograr objetivos comunes o propios, los actores, con cierta racionalidad, voluntariamente delinean estrategias de interacción al necesitarse entre sí por su interdependencia de recursos; a la par, ello implica una oportunidad de influir en las 
políticas públicas y por lo cual tratan de orientar sus interacciones hacia sus propias preferencias de políticas.

Al pensar a las redes de política pública como una opción para propiciar la cooperación se reconoce la idea básica del enfoque de la acción colectiva: «gente que actúa conjuntamente en orden de llevar acabo objetivos deseables, inclusive si ellos no son igualmente esperados» (Carlsson, 2000: 510) o compartidos por los actores dado que no puede haber unicidad de objetivos dentro de una acción organizada (Crozier y Friedberg, 1980), como lo es una RPP.

Se reconoce el carácter interactivo y las interdependencias o interrelaciones instrumentales que ligan a las partes involucradas en las redes de política pública, ya que sus procesos interactivos y su desempeño vienen determinados por más de un actor que posee sus propios intereses y estrategias para influir en la construcción de las decisiones públicas. Pero, así como su conformación no es fortuita o espontánea, la interdependencia tampoco es similar para todos los actores en cuestión porque implica un mínimo de integración y relaciones instrumentales (intercambios de recursos), lo cual pone a flote las situaciones o relaciones de poder coexistentes en el seno de las redes de política pública. Ahondar al respecto es materia de lo siguiente.

\subsection{El poder en las redes}

Según Mouffe (2000) y Knoke (1990), las relaciones sociales difícilmente son concebibles sin algún tipo de dominio provisional, sin exclusiones y desigualdades, sin antagonismos, debates y polémicas, sin conflictos y tensiones entre los agentes en cuestión. Esto denota importantes implicaciones para el estudio de los procesos de gobernanza como lo son las redes de política pública, dada la naturaleza social de las relaciones que en ellas se suscitan, conduciendo a que se considere obligatoriamente que, al hablar de redes, no se está tratando de un espacio estable y de relaciones solidarias per se, donde el poder está distribuido igualitariamente en una estructura no-jerárquica. Sino todo lo contrario, las redes se asemejan más a ámbitos de relaciones de poder con estructuras poco horizontales, debido al estado asimétrico de la dependencia de recursos y porque las relaciones de intercambio siempre favorecen más a una de las partes, denotándose situaciones de dominio.

Así, el denominador común de todas las manifestaciones de poder (cualesquiera que sean sus fuentes, legitimación, objetivos y métodos de ejercicio), implica siempre la posibilidad para algunos individuos o grupos de actuar sobre otros y para lo cual habrá que relacionarse, ya que para actuar sobre un tercero, es necesario entrar en relación con él, constituyéndose ahí una relación de poder. De ahí que lo esencial del poder es su carácter de relación y no un atributo de los actores. Además, se trata de una relación situada y, por lo tanto, contingente, tanto a los actores como a la estructura en la cual actúan (Crozier y Friedberg, 1980).

Pero ¿cómo analizar una relación de poder? y ¿por qué hay ciertos actores que ocupan una posición privilegiada en las decisiones públicas? Para Crozier y Friedberg implica considerar (a) los recursos de que dispone cada actor que le permiten ampliar su margen de dominio y (b) establecer los criterios que definen la pertinencia de esos recursos. 
(a) Se reconoce que hay una variedad de recursos de los que puede disponer un actor por su situación social, económica, cultural, etc. Tener en cuenta la diversidad de recursos hace posible introducir en el análisis las desigualdades (asimetrías) entre actores. Al respecto, Themudo (2000: 6) señala que si bien no existe una regla específica para determinar qué sí y qué no es un recurso, propone una definición muy cercana a la que ofrece la Real Academia al indicar que «si algo ayuda a lograr un objetivo, ya sea en el presente o futuro, entonces se trata de un recurso»».

A la luz de lo señalado, se entiende como recursos a los insumos que son relevantes en sí mismos para el funcionamiento de la red y porque permite a los agentes la consecución de sus objetivos. Luego, si bien Crozier y Friedberg (1980) hablan de un abanico de recursos, muy poco avanzan por concretizar o describir la variedad de recursos a los que se refieren. Para salvar esta situación se recurre a la propuesta de Edwards y McCarthy (2004), quienes en sus aportaciones a la Teoría de Movilización de Recursos sugieren que éstos pueden ser: morales, humanos, organizacionales, materiales y culturales.

(b) Continuando en la misma lógica puesta por Crozier y Friedberg, problematizamos acerca de ¿cuáles son los criterios que definen la pertinencia de esos recursos y su carácter más o menos movible? Esto obliga a cambiar de orientación. Se trata de prestar atención a las limitaciones estructurales al poder que los actores tratan de ejercer entre sí al movilizar y condicionar sus recursos. Es decir, el análisis de una relación de poder debe ubicarse dentro de las limitaciones que impone la red (esto es, las acotaciones impuestas por la estructura y las reglas) y que influyen en la restricción de la libertad de acción, racionalidad y voluntad de los individuos y grupos.

Aceptando el punto de las limitaciones estructurales de la red es que se considera que ella genera sus propias fuentes de poder. De tal forma, dichas fuentes definen la conveniencia de los recursos y con ello el margen de dominio y negociación del actor, por cuanto que los recursos disponibles no son igualmente pertinentes ni movilizables debido a su criticidad y escasez (Pfeffer y Salancik, 1978); ello en correspondencia a los objetivos de la RPP, implica que en alguna política pública se valoraran más unos recursos y se descartarán otros, según los objetivos concretos de la acción y de los intereses de los actores público-sociales, privado-lucrativos y gubernamentales. De ahí la importancia de la escasez y criticidad de los recursos. En estos términos:

- Críticos son aquellos recursos sin los cuales los actores sociales, privado-lucrativos y gubernamentales no pueden desarrollar sus actividades y funcionar según su misión y visión. Más aún, tienen una importancia primordial para el desarrollo de la acción colectiva que los reúne.

- Un recurso es escaso en la medida en que no se encuentra fácilmente disponible en el ambiente en el que se desarrolla la política pública.

En esta lógica, es que se sugiere que los grupos o los actores que controlan aquellos recursos críticos son los que tienen más poder, al permitirles ampliar su margen de dominio y negociación en la red. Así, la propuesta de Pfeffer y Salancik (1978) acerca de la criticidad y escasez de los recursos, se complementa con lo que propone la Teoría de Movilización de Recursos de Edwards y McCarthy (2004). Cruzar ambas propuestas permite una tipología de los recursos y caracterizarlos como críticos o escasos. 
Teniendo en cuenta las propuestas de Crozier y Friedberg (1980), Pfeffer y Salancik (1978) y Edwards y McCarthy (2004), se considera que los actores que portan o controlan los recursos críticos (sean morales, humanos, organizacionales, culturales o materiales), son quienes con mayor facilidad priorizarán sus preferencias en los objetivos de la decisión pública a fin de que su participación en la red les reditúe, lo cual puede leerse como una manera de aventajar en la construcción de las decisiones colectivas. De hecho, aplicando las ideas de Crozier y Friedberg (1980), los actores difícilmente alcanzan sus intereses más que por el ejercicio del poder que se suscita en las relaciones sociales (como las que se dan en las RPP), al tiempo que no pueden ejercer su poder más que cuando persiguen objetivos comunes a través de acciones organizadas según se discute a continuación.

\section{Las redes de política pública como constructos de acción colectiva y las implicaciones de su estructura}

$\mathrm{Al}$ suponer a las redes de política pública como posibles alternativas para propiciar la cooperación entre una de serie de actores relativamente autónomos, con sus recursos y capacidades particulares, implica reconocerlas como constructos que tratan de articular e integrar comportamientos divergentes (y posiblemente contradictorios) instituyendo una estructuración humana o un mínimo de organización del campo de acción social. En consecuencia, las posibilidades de cooperación de los actores en mucho se relacionan con la estructura o composición organizativa de las redes de política pública.

Los partidarios de las redes de política pública (entre ellos Carlsson, 2000) argumentan que a fin de una mayor comprensión de cómo las políticas se crean en la sociedad se debe investigar las estructuras emergentes para atender los asuntos públicos en lugar de centrarse en las estructuras formales, las autoridades políticas, sus decisiones y programas. Siguiendo a Jones, Hesterly y Borgatti (1997), contemporáneamente, hay formas de cooperación que no derivan de las estructuras de autoridad o de las reglas formalizadas dado que si bien esos mecanismos definen las relaciones entre ciertos actores participantes no lo hacen con todos los involucrados ya que puede haber relaciones no especificadas entre participantes no reconocidos por la estructura formal pero que se vinculan con alguno o algunos participantes reconocidos formalmente. Así, las RPP implican actividades conjuntas de actores variablemente autónomos y relativamente organizados en una estructura formal o no en torno a un sector de política pública.

De tal forma que si muchos procesos de acción colectiva resultan contraproducentes a los objetivos de quienes los emprenden, no se debe exclusivamente a las propiedades de los problemas, características de los individuos ni a sus estrategias no-cooperativas, sino que también tienen que ver las propiedades de la estructuración del campo de acción o de los constructos de acción colectiva mediante los cuales se trata de lograr la cooperación. Es decir, hay cierto reconocimiento a la incidencia de la conformación estructural de las redes de política pública en el proceso y los resultados de la política.

De tal forma, los resultados de políticas públicas y los patrones de interacción junto con las estructuras de las redes de política se pueden ubicar en una serie de conexiones 
lógicas, en el sentido que dicha relación interactiva «entre redes y actores define, da forma, interpreta y reinterpreta los resultados de las políticas públicas. Al mismo tiempo, los actores participantes interpretan, reinterpretan y limitan a la red. Por lo tanto, existe una compleja relación entre la red (estructura) y la agencia» (Evans, 1998: 253).

En sintonía con lo discutido se construye una relación lógica entre las interacciones de los actores y las posibilidades de cooperación entre ellos, a partir de reconocer ciertas propiedades estructurales de las redes de política pública. Tal propuesta se robustece con los avances que ha logrado el análisis formal del estudio de redes sociales, desde donde ha cobrado importancia la composición estructural de las redes sociales (Knoke y Yang, 2008; Hanneman y Riddle, 2005; y Knoke, 1990). Es decir, hay una relación directa entre lo que propone Crozier y Friedberg (1980) y lo que desde el análisis de redes sociales se prescribe, al señalar que la estructura de la red (estructuración del campo de acción para Crozier y Friedberg) impone oportunidades y constreñimientos a la acción de los actores a fin de favorecer objetivos propios y colectivos.

\subsection{La estructura de la red de política pública como factor incidente}

Considerando la composición de la acción organizada como variable clave para entender los procesos cooperativos (Crozier y Fierdberg, 1980; véase también a Olson, 1965), y teniendo en mente aportaciones más actuales como las de Sandström y Rova (2010) y Sandström y Carlsson (2008), así como retomando los avances que se han logrado desde el enfoque del análisis de redes sociales (Knoke y Yang, 2008; Wasserman y Faust, 1994; y Knoke, 1990), es que establezco los atributos de las redes de política pública en términos estructurales.

Considérese que al interior del análisis de redes sociales, la idea de que las estructuras de las redes sociales son importantes variables explicativas es aceptada. Los proponentes argumentan que la estructura, la cual es referida a cuántos actores se encuentran conectados al interior de la red y cómo se organizan sus relaciones, elucida las características estructurales subyacentes de las relaciones más estables (Sandström y Carlsson, 2008).

En esta tesitura, Sandström (2008) considera que la estructura entre las relaciones de los actores y su localización en la red tienen importantes consecuencias conductuales, de percepción, de actitud para las unidades individuales, y para el sistema como todo (aseveración que ya había sido planteada por Crozier y Friedberg, 1980). Por eso, es posible presumir que los patrones de las relaciones favorecen o restringen la cooperación en el sentido de que «...el éxito de una política no depende únicamente de su contenido y cualidades de los actores involucrados, también de la red como un todo. Por eso, algunas redes quizá son asumidas para ser más efectivas que otras en lograr acciones colectivas... (Sandström, 2008: 42).

En concreto, las redes de política pública como constructos de acción colectiva que integran y articulan comportamientos -si bien voluntarios-, instituyen al mismo tiempo cierta estructuración humana y de poder, i.e. la estructura de la red (con ciertos atributos o cualidades) que incide en la manera de darse la interacción entre los actores miembros, siendo unas más favorables para la cooperación que otras. 
Por lo indicado, cobra sentido considerar que las redes de política pública son más que agregados de individuos racionales con ciertas especificidades, sino que las redes como estructuras organizativas implican recursos en juego, relaciones de poder y reglas en uso. Aspecto que no debe darse por sentado. De ahí, la importancia de considerar a las RPP a partir de reconocer sus atributos en términos estructurales.

\subsection{Pensando a las redes de política pública partiendo de sus atributos estructurales}

El reconocimiento de las redes como estructuras organizativas ha implicado que sean estudiadas a través de lo que se identifica como el análisis formal de redes. Desde esta perspectiva, se asume a las redes como estructuras que «atribuyen papeles, recursos y capacidades» que «afectan tanto la manera en que se comportan los grupos como los resultados de las políticas» (Evans, 1998: 250). De tal forma, el foco de atención no son los agentes en sí mismos sino las posiciones que ocupan, sus relaciones y conexiones entre ellos.

En tal tesitura, es menester tener presente que la importancia del análisis de redes sociales descansa en tres supuestos acerca de los patrones de las relaciones y sus efectos: primero, «las relaciones estructurales con frecuencia son más determinantes para entender los comportamientos observados que atributos personales como edad, género, valores e ideología»; segundo, «las redes sociales afectan las percepciones, creencias y acciones a través de una variedad de mecanismos estructurales que son socialmente construidos por relaciones entre entidades»; tercero, «las relaciones estructurales son procesos dinámicos. Las redes no son estructuras estáticas, continuamente cambian a través de las interacciones entre las personas, grupos u organizaciones que las constituyen» (Knoke y Yang, 2008: 5-6).

Como complemento al análisis de redes sociales, las inferencias y algunas conclusiones de los teóricos del capital social coadyuvarán a identificar las cualidades estructurales de las redes de políticas, así como a justificar la importancia que adquieren para comprender y explicar los procesos cooperativos (en contextos de política pública) y sus resultados.

Por lo desarrollado, y considerando los trabajos de Luna y Velasco (2009) y Sandström (2008 y 2004), el ARS ofrece herramientas analíticas y operacionales valiosas para el estudio de las redes de políticas públicas, pero en sí mismas no permiten llevar a las RPP más allá de su sentido descriptivo, por ello se ha hecho necesario tomar como eje articulador la propuesta de Crozier y Friedberg (1980), en la cual se reconoce la importancia de las propiedades de la estructuración de los constructos de acción colectiva para entender los procesos de cooperación (es decir, las estructuras sí importan), recurso teórico que es consistente con lo que desde la literatura de redes de políticas públicas se ha señalado (Klijn, 1997) y ampliamente compatible con los preceptos del ARS (Knoke y Yang, 2008). Especialmente, Crozier y Friedberg (1980) contribuyen a explicar a los actores, sus interacciones, las posibilidades de intercambio de recursos entre ellos, las asimetrías de recursos, y sus posiciones estructurales, a fin de perfilar la conexión lógica entre ciertos atributos estructurales de las redes, patrones de interacción y su incidencia en la cooperación. 
Adicionalmente, las aportaciones de los teóricos del capital social permiten complementar dicha conexión lógica ya que básicamente el CS se enfoca en «cómo las relaciones compartidas entre los sujetos se convierten en un dispositivo productor (o inhibidor) de beneficios sociales» (González, 2009: 1732) u objetivos de alcance social.

De esta forma y aunque el concepto de capital social no ha sido examinado explícitamente en lo presente, su discusión breve facilitará concretar ciertas características o atributos de la estructura de la red. En estos términos, Coleman (1988) como uno de los pioneros en la investigación sobre el CS influiría en Burt (1997) y en Putnam (1993), teniendo sus propuestas el común denominador de identificar en el capital social estructuras relacionales, funcionales e institucionales.

De tal modo, retomando a Burt $(2000,1994$ y 1997) y a Coleman (1990 y 1988), Sandström (2008) indica que hay dos elementos básicos en los cuales coinciden las líneas teóricas y de investigación sobre el CS. Primero, el capital social permite llevar a cabo ciertos fines que en ausencia de éste no serían posibles. Segundo, esta ventaja, se supone, brota de la estructura social, ya que las personas que están mejor conectadas logran mejor desempeño. De ello, se puede inferir que el capital social refiere a las características de la organización social que facilitan la coordinación y cooperación para beneficios mutuos (Putnam, 1995). Este reconocimiento que se da a la estructura organizativa como posible determinante de que se propicien o no procesos colectivos de acción, ha recibido la atención de autores como Burt (2000 y 1997), Coleman (1990 y 1988) y Granovetter (1973).

La contribución seminal de Granovetter (1973) radica en dilucidar que las redes sociales trabajan a partir de estructuras relacionales de intercambio siendo el capital social una manera de entender un tipo de estas relaciones. Dicha proposición la deriva de entrecruzar los avances teóricos del análisis de redes sociales y la dimensión del capital social. En su trabajo, Granovetter (1973) explica la manera como los sujetos aprovechan o no las oportunidades potenciales de las relaciones sociales informales y para lo cual utiliza el concepto de lazos fuertes y débiles. Los primeros representan las relaciones cercanas (parentescos o vínculos de amistad íntimos). Los segundos comprenden la combinación de cercanía social, multiplicidad de relaciones y contactos frecuentes. En este sentido, para Granovetter (1973) los lazos fuertes son menos importantes que los lazos débiles para sostener la acción colectiva; es decir, no son fuente principal de capital social. Esto pasa, pues las redes personales de lazos fuertes son redes densamente cerradas, aquí la información compartida es muy pobre y poco tendiente a capturar novedades por fuera de ese entorno. Esta característica es positiva, por ejemplo, para difundir alguna innovación o generar cohesión, pero muy negativa al momento de buscar recursos alternativos y producir las principales formas de capital social.

Se ha avanzado la propuesta de Granovetter a través de los trabajos de Burt (2000) y Coleman (1990), quienes se han focalizado en estudiar las propiedades de las relaciones al interior de las redes, haciendo uso de los conceptos y herramientas del análisis de redes sociales. Por lo que, desde las propuestas de Coleman y Burt, la investigación de configuraciones de la red que promuevan el capital social es el principal objetivo, tratando de identificar las cualidades de la red que pudiesen explicar por qué ciertos desempeños individuales o de grupo son mejores que otros. 
En este sentido, Burt (2000) ha notado que primariamente son dos los atributos estructurales relevantes para establecer la relación entre la red y el capital social, por un lado se trata de la integración de los miembros de la red y por el otro su heterogeneidad o agujeros estructurales. Así, «el argumento central que supone la integración señala que el capital social es generado por agentes fuertemente interconectados»». Mientras que «el argumento de los agujeros estructurales indica que el capital social es propiciado por las conexiones que los agentes pueden establecer con otros segmentos de otro modo desconectados» (Burt, 2000: 1).

De tal forma, mientras que la integración se refiere a la conectividad de la estructura local y uniformidad interna de los miembros de la red, los agujeros estructurales o heterogeneidad indica principalmente la diversidad y la estructura global de la red (esto es, la relación de la red en cuestión con otras redes).

\subsubsection{La integración de la red}

Este primer atributo o cualidad se asocia con la idea de Coleman (1990) que plantea que un alto nivel de interconectividad de la red propicia que la calidad de la comunicación entre los integrantes se mejore, se generen normas comunes y las posibilidades de comportamientos oportunistas se restrinjan, de hecho se trata de una aseveración muy cercana a las ventajas de los grupos pequeños (privilegiados) que indicaba Olson (1965), en el sentido de que en las redes bien integradas nadie puede escapar de la atención de los demás, propiciando que los integrantes procuren mejorar o mantener su reputación a efecto de ser identificados como sujetos con credibilidad y confianza (Burt, 2001).

Se puede asumir que «una red bien conectada promueve la acción colectiva y fortalece las capacidades organizativas de la red de política pública. En esta misma lógica, la formación de instituciones, así como la creación y mantenimiento de las normas se mejoran dentro de tales redes, con ello mejorando el proceso de la política» (Sandström y Carlsson, 2008: 508).

\subsubsection{Heterogeneidad estructural}

Cuando el nivel de análisis es al interior de la red, los agujeros estructurales indican aquellas secciones de la red desconectadas entre sí (Burt, 2000). Pero si el nivel de análisis es de la red con su ambiente implica reconocer sus vínculos con terceras partes (sean individuos, organizaciones u otras redes) o los agujeros estructurales globales; esto supone ventajas estratégicas al facilitar el acceso a recursos nuevos, al iniciar innovaciones, al agregar valor a la red a partir de sus vínculos globales, y al integrarse a otras redes. Por ello, las redes ricas en agujeros globales (o altamente heterogéneas) tienen más posibilidades de: acceder a información novedosa, establecer alianzas estratégicas, sinergizar recursos, disponer de más alternativas para sus decisiones estratégicas (reducir riesgos), ser más flexibles para adaptarse a cambios exógenos e innovadoras (García, 2009). 
De acuerdo a esto, «redes que expanden una variedad de agujeros estructurales son redes ricas en capital social. El argumento esencial inherente es que el acceso de un individuo a los recursos está determinado por las características de las relaciones sociales de esa persona» (Sandström y Carlsson, 2008: 508). Por lo que entre más relaciones tenga un actor (más allá de las que establezca en la red) y entre más diversas sean respecto a las relaciones de otro actor, más posibilidades de expandir agujeros estructurales globales. Esto es, se está pensando que entre más heterogéneos sean los actores que se integran a la red más posibilidades de que la red cuente con más recursos y más variedad de ellos.

Llegados hasta aquí, es perceptible que ambos atributos (integración y heterogeneidad) parecieran correr en sentido contrario, pero de cualquier forma su utilidad para estudiar a la estructura de la red se fundamenta en la síntesis que hace de ellos Burt (2000) y que retoman Sandström y Carlsson (2008) al indicar que si a través una alto nivel de heterogeneidad se agrega valor a red, la integración de la red es crítica para aprovechar el valor agregado.

En consecuencia, al hacer perceptible y otorgar un sentido lógico a la relación entre la red de política pública a través de dos de sus atributos estructurales (integración y heterogeneidad) y las interacciones; tempranamente, se advierte que es muy probable que la variabilidad de la integración y heterogeneidad influya en las interacciones entre los actores, lo cual implica problematizar acerca de dicha relación.

\section{Las interacciones en la red: su carácter variable}

Anteriormente se ha indicado que si las consecuencias de la acción colectiva se contraponen a las voluntades de los actores, se debe también, entro otros aspectos, a factores estructurales, y para lo cual se han sugerido dos atributos estructurales básicos de las redes (el nivel de integración y heterogeneidad). Esto implicando reconocer que la relación dialéctica entre las decisiones deliberadas del actor y la estructura ocurre mediante las interacciones de los actores (Granovetter, 1992), mismas que según se expone a continuación se caracterizan por ser variables, siendo unas más propicias para la cooperación que otras.

Desde la postura de Granovetter (1992) se acepta la relación entre la estructura y las interacciones. Esta asociación traída al estudio de las redes de política pública conduce a reconocerlas como entidades organizativas o constructos instituidos para propiciar procesos de cooperación como los que conllevan las políticas públicas en las cuales intervienen agencias gubernamentales, actores público-sociales y privado-lucrativos. Y, al decir que no únicamente un actor es el que interviene en la política, supone un mínimo de interacción entre varios actores que toman parte en las políticas públicas como una manera de participar en los asuntos públicos.

En esta tesitura, Teisman (2000) ha propuesto un modelo interactivo para analizar los procesos de política pública. Así, en contraste con al modelo de fases, aquí (en el modelo interactivo) se asume que los problemas y soluciones no son ligadas a un solo actor (al responsable político) y no son por lo tanto fijadas en un momento en particular. Muchos actores están involucrados en la formulación e implementación de la polí- 
tica, y ellos introducirán sus percepciones acerca del problema, posibles soluciones y juicios políticos.

El modelo interactivo, expone Teisman (2000), implica considerar a las políticas públicas como sucesiones de decisiones cruciales que los agentes generan en torno al problema en mano, pero en las cuales no todos participan o se involucran de manera homogénea (ver esquema 1). De esta manera, si nos enfocamos a las estructuras de las redes, se advierte la estabilidad relativa de dichas estructuras considerando el conjunto de instituciones que las soportan, pero no así de las interacciones que de ella se derivan, ya que desde el modelo de Teisman en los distintos eventos de la decisión pública se asume que hay actores que entran y salen, se vinculan o desvinculan entre sí.

Para un mismo problema o asunto público, si bien la estructura de la red que se constituye en su atención probablemente tiende a ser estable, de eso no se deriva que las interacciones lo sean ya que los actores miembros se insertan en una dinámica en la cual desarrollan esquemas interpretativos y estrategias dependiendo su posición dentro de la red (o en la estructura), del sistema de reglas que aplique, según su percepción acerca del asunto público en mano y al amparo de los recursos que controlen o aporten.

\section{Esquema 1. Modelo interactivo para el análisis de la política pública ${ }^{1}$}

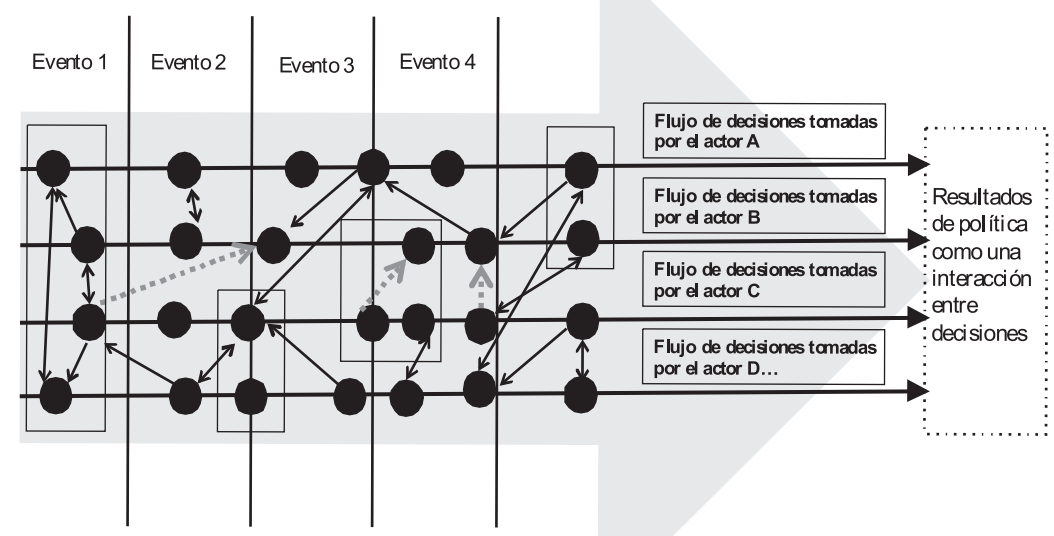

Fuente: elaborado a partir de Teisman (2000).

${ }^{1}$ La flecha gris de fondo es el proceso de decisiones, los puntos negros indican las decisiones adoptadas por los distintos actos, y el resultado de la política se deriva de la interacción entre las decisiones (construidas sobre las decisiones de los demás $<$ flechas negras más pequeñas $>$, la anticipación de las decisiones futuras $<$ flechas grises $>$ y pactando resultados $<$ rectángulos $>$ ) (Teisman, 2000). 
De hecho, aplicando el supuesto de que en las redes de política pública la estructuración del comportamiento humano difícilmente será horizontal por la asimetría de dependencia de recursos entre los miembros que las integran (Sandström, 2008) y porque no todos los recursos tienden ser movilizables dada su pertinencia (determinada principalmente por su criticidad) para una política pública específica; así como, por las imbricaciones de esto con las relaciones de poder, se asume que es muy poco probable que en las redes de política pública se susciten interacciones homogéneas y estables entre sus miembros.

Más bien, al decir interacción implícitamente, se dice relación y, como ya se ha indicado, es una de las características básicas del poder en su carácter relacional, donde una de las partes es más favorecida que las otras. Se trata de una relación donde un actor público-social, privado-lucrativo o el gobierno puede sacar ventaja, donde existen equilibrios de poder y asimetrías, lo cual conduce a considerar la existencia de algún actor dominante, pero donde todo ello tiene una justificación estructural y es contingente.

Más concretamente, se está hablando de diferentes formas de interacción como equilibrios entre las estrategias de poder de los actores en juego y donde uno de ellos domina. De eso, es posible presumir: (a) que al decir interacciones, debemos señalar una variedad de patrones interactivos más que denotar un complejo interactivo homogéneo y estable, (b) y que no todas las interacciones favorecen a la cooperación.

Por ello, es imprescindible diferenciar o distinguir algunos modos (patrones) de interacción potenciales que se pueden suscitar en una red de política pública, y para lo cual será necesario indicar previamente los mecanismos de vinculación y las funciones organizativas que se suscitan en la red.

\subsection{Mecanismos de vinculación y funciones organizativas claves en las redes a modo de identificar los patrones de interacción}

Atrás se ha sugerido que las conexiones entre los actores miembros de una red como resultado del intercambio de recursos, exige la identificación de distintos modos de interacción, y que se propone entenderlos como las relaciones contingentes y relativamente estables entre dos o más actores (Vázquez, 2010). Pero para precisar esos patrones de interacción es necesario señalar los mecanismos de vinculación que pueden suscitarse entre los integrantes de la red.

\subsubsection{Mecanismos de vinculación}

Un mecanismo de vinculación es el componente a través del cual dos o más actores se engarzan, desarrollan relaciones de dependencia e interactúan. La importancia de reconocer los mecanismos de vinculación entre los actores integrantes de una red, radica en que éstos permiten observar cómo se relacionan entre ellos y entre quienes. En este sentido, Natal (2007) propone observar los mecanismos de vinculación a partir de considerar que éstos pueden ser estructurales u operacionales. Utilizando esta herramienta conceptual para nuestro propósito considérese que: 
- los mecanismos estructurales implican reconocimiento y vinculación formal entre los integrantes, permitiéndoles influir en las características de la estructura, toma de decisiones, desempeño y elecciones de la red.

- los operacionales implican vínculos informales que se desarrollan para apoyar un tipo específico de actividad o proyecto y para establecer alianzas, sin que impliquen algún tipo de membresía o relación formal al interior de la red.

En tal tesitura, estudiar los patrones interactivos entre los actores o integrantes de una red, reconociendo sus mecanismos de vinculación, se justifica por cuanto «...muchas o la mayoría de las propuestas de política bien diseñadas nunca llegan a ser efectivas. La razón es que la política pública usualmente no es producida por un actor unitario con control adecuado sobre todos los recursos requeridos para una acción. Más que eso, es probable que el resultado derive de la interacción estratégica entre varios o muchos actores, cada uno con su propio entendimiento de la naturaleza del problema y factibilidad de una solución en particular, con su propio interés y preferencias normativas, cada uno con sus propias capacidades o recursos de acción que quizá empleen para afectar el resultado de política pública» (Scharpf, 1997: 11).

En consecuencia, se trata de prestar atención a las interacciones entre los actores; por cuanto, las políticas no únicamente son decididas; también implican a personas que, mediante algún mecanismo de vinculación, interactúan desarrollando funciones organizativas al interior de la red.

\subsubsection{Funciones organizativas}

Siguiendo a Mintzberg (1979), se asume que las redes de políticas públicas en orden de llevar a cabo actividades específicas, desarrollan patrones de división del trabajo. Por eso, aunque las redes de política pública como estructuras organizativas no comparten necesariamente las características de las organizaciones formales, ellas pueden ser percibidas como la suma de funciones organizativas, y pueden ordenarse en cuatro: definición del problema, priorización (decidir qué solución deberá ser adoptada), movilización de recursos y evaluación (Carlsson, 2000).

En dichas funciones quienes integran una RPP interactúan, redefinen su posición estructural, entran y salen de la red, y toman decisiones cruciales. No obstante, investigaciones (Sandström y Rova, 2010; Sandström y Carlsson, 2008) que han utilizado las funciones organizativas mencionadas para estudiar los procesos de decisiones públicas, han señalado la importancia relativa de dos de ellas: la priorización y la movilización de recursos.

Así es como los actores participantes en una red de política pública, una vez identificado el problema o asunto público sobre el cual se interesan y en torno al cual deciden incidir, hay dos funciones primordiales para que el proceso de la política pública proceda: la priorización y la movilización de recursos. La priorización de las líneas de acción es fundamental por los numerosos caminos alternativos a seguir respecto a cómo mejorar la situación presente y motivo de la política pública, además de que cada alternativa implica ventajas o desventajas para los objetivos particulares de cada inte- 
grante de la red. Acto seguido de la priorización, los recursos necesarios deben ser movilizados; por lo cual, al amparo de los recursos disponibles cada alternativa será viable o no.

En esta tesitura, cada una de estas dos funciones vitales tiene varios atributos importantes. Así, respecto a la priorización habría que tener en cuenta tres de ellos: (a) el tiempo relativo que llevó a los actores a priorizar una acción sobre las alternativas, (b) la legitimidad ${ }^{2}$ entre los actores de la decisión frente a otras opciones y (c) la metodología para tomar decisiones. En cuanto a la movilización de los recursos habría que considerar: i) el grado de interdependencia de recursos entre los actores y i) el mecanismo de vinculación entre ellos.

\subsection{Patrones de interacción y su relación con la estructura de la red de política pública}

En base a los elementos descritos es posible proponer una escala de variación de los patrones de interacción; esta propuesta cobra sentido si consideramos que entre los actores miembros de una red de política pública, altos niveles de integración favorecen a la función de priorizar, esto es: de que en el corto tiempo se adopten las soluciones y que las mismas sean las más aceptadas entre otras alternativas. En tanto que entre más heterogénea sea la red, implica que el proceso de movilización de recursos será menos problemático, ya que habrá más cantidad de ellos y serán más diversos.

Basado en lo anterior, propongo una escala de variación de las interacciones, a través de patrones de interacción: asociación, acciones combinadas y actividades superficiales. ${ }^{3}$ De este modo:

A. En una interacción a nivel superficial, el proceso de priorización implica que el tiempo en el cual se han preponderado las decisiones es relativamente alto, y aun así la legitimidad de las decisiones a emprender es baja frente a otras alternativas. La metodología para la toma de decisiones es la consulta y el voto. En cuanto a la movilización de recursos el grado de interdependencia entre los actores es baja, hay recur-

2 Uso aquí el término legitimidad en un sentido restringido. Así, una solución es legítima porque se juzga conveniente de ejecutar (Méndez, 2000).

${ }^{3} \mathrm{Al}$ respecto conviene aclarar algunos puntos: (a) El propósito es ofrecer una primera aproximación a ciertos parámetros de las interacciones que suceden en las redes de política pública. A pesar de que se trata de un elemento básico y ampliamente reconocido en el enfoque de Redes de Política Pública, su estudio detallado y su tratamiento como variable es escaso. (b) No se trata de una escala omnicomprensiva en la cual se amolden todas las formas de interacción de las redes de política pública ya que la propuesta es construida en términos ideales. (c) La propuesta de ordenar o más bien proponer tres niveles o patrones de interacción, es deudora de las aportaciones de Natal (2001). De igual, reconozco las contribuciones de Teisman y Klijn (2002) y Kickert, Klijn y Koppenjan (1997) quienes señalan los roles que los actores público-gubernamentales y privados pueden asumir en distintos modos de darse los acuerdos de colaboración entre ellos; así como, discuten la perspectiva interaccionista de las redes de políticas, respectivamente. También, han sido valiosas las discusiones de Luna y Velasco (2009) acerca de las propiedades de las interacciones suscitadas en las redes de política pública como sistemas asociativos complejos. 
sos de los cuales se pueden prescindir para que proceda la política pública. Se trata más bien de una relación de consulta. Este nivel de interacción implica que la priorización y movilización de recursos se puede dificultar y llegar a ser problemática. Por otra parte, la vinculación entre los actores que interactúen a nivel superficial se da principalmente a través de mecanismos operacionales.

B. La interacción a nivel de acciones combinadas describe que la priorización de las decisiones sucedió en un tiempo moderado y las decisiones a emprender no son del todo legítimas. La metodología para tomar decisiones es la negociación. Respecto a la movilización de los recursos el grado de interdependencia es media; esto denota que los participantes se involucran en escenarios tempranos o iniciales de la política pero desarrollan separadamente sus funciones. Así, este tipo de interacciones implican que los actores, al vincularse, presentaran reticencias para movilizar sus recursos conjuntamente y más bien actúan separadamente. Adicionalmente la vinculación puede darse a través de mecanismos operacionales como estructurales.

C. Establecer interacciones a nivel de asociación implica que la priorización de las decisiones sucede en corto tiempo y ampliamente caracterizadas por su legitimidad. La metodología para la toma decisiones es la deliberación. Aquí la movilización de recursos es más fluida ya que la interdependencia es alta, los recursos de los actores son imprescindibles para los fines particulares y para favorecer los objetivos colectivos que se enmarcan en la decisión pública. Esto configura una relación permanente de intercambio de recursos. Además, el mecanismo de vinculación que predomina es del tipo estructural.

Llegados hasta aquí ya es posible perfilar cómo la estructura de la red afecta a las interacciones y en qué sentido. Así las cosas, una alta heterogeneidad e integración estructurales propician interacciones a nivel de asociación entre los actores miembros de la red. Una heterogeneidad e integración media implican interacciones a manera de acciones combinadas. En tanto que, cuando existen niveles bajos de heterogeneidad e integración en términos ideales se propician interacciones superficiales.

En tales términos, se puede presumir que las redes de políticas públicas sí importan y reconocerlas a través sus atributos estructurales implica una hebra lógica que conduce a plantear que hay interacciones que pueden ser más propicias para detonar procesos cooperativos que otros. De tal forma que cuando la red de política pública se caracteriza por una alta heterogeneidad e integración estamos hablando de un acción organizada cuya interacción entre sus integrantes será a nivel se asociación implicando mayor propensión a que cooperen entre ellos mediante procesos de interdependencia; en los cuales, si bien habrá algún actor dominante, la posición de dominio puede ser acordada entre los participantes y depuesta según las características y tiempos de la política pública en mano.

Cuando la red se caracteriza por una mediana integración y heterogeneidad, se suscitarán interacciones a modo de acciones combinadas entre los actores de la red, implicando procesos no totalmente cooperativos y jerarquías no del todo acordadas según los roles que asuman los integrantes de la red. Y ya en el último de los casos, cuando se suscitan interacciones superficiales de darse la cooperación, ella no es del todo voluntaria sino más bien se genera mediante una jerarquía preestablecida de modo que las unidades de mando tienden a ser concentradas en una coalición dominante cuya 
vinculación con los otros actores de la red se da principalmente a modo de legitimar sus decisiones y acciones, y no necesariamente para que aporten sus recursos y se propicien procesos de cooperación.

\section{Conclusiones}

Este documento asume a las redes de políticas como una expresión de los procesos contemporáneos mediante los cuales atienden los asuntos públicos gobierno y sociedad, actores privado-lucrativos y público-sociales y/o de la sociedad civil.

Los desarrollos expuestos provocan ciertas hipótesis que establecen un itinerario de investigación en redes de políticas públicas, gran parte del cual implica acortarse mediante estudios de caso que aporten evidencia empírica a modo de evaluar la propuesta aquí esbozada.

Por lo pronto, este trabajo ha avanzado la discusión en torno a las redes de política pública a modo de comprender de manera más profunda ciertas expresiones contemporáneas del tratamiento y atención de los asuntos públicos en el marco de lo que identifican como gobernanza. Para ello, he considerado aportaciones que empíricamente establecen alguna relación entre la estructura de una red de política pública respecto a sus capacidades organizativas y los resultados, deseados o no, que se propician (véase a Berardo, 2009; Percival, 2009; Lewis, 2005; Howlett, 2002; y Provan y Milward, 1995); con lo cual, existe un punto de partida para contrastar la propuesta de este trabajo, que a diferencia de los citados, se propone vincular a la estructura de la red y la cooperación a través de las interacciones que se suscitan al interior de la red, en el sentido de que hay interacciones más propicias para la cooperación que otras.

Así, según sea la variabilidad de la heterogeneidad e integración se propician tres tipos de interacciones. En términos de la argumentación propuesta, cuando la estructura de la red de política pública se caracteriza por una alta heterogeneidad e integración, hay grandes probabilidades de que los actores miembros o participantes en la red desarrollen interacciones asociativas y lo cual implica altas posibilidades de que se detonen procesos cooperativos en torno a objetivos colectivos. En cambio, si las cualidades estructurales son de mediana heterogeneidad e integración, las interacciones serán en términos de acciones combinadas, ello traduciéndose en procesos no totalmente cooperativos. Finalmente, cuando una red se caracteriza por su baja integración y heterogeneidad, las interacciones entre los miembros son superficiales y con minúsculas posibilidades de cooperación.

En términos de las posibilidades de desarrollo empírico de la propuesta desarrollada, estas pueden asumir a la RPP como un todo y observar sus cualidades estructurales, y a partir de ahí evaluar el tipo de interacción que se ha suscitado y predominado en un tiempo determinado. O bien, se podrían observar que hay distintas formas de interacción en una red de política en un momento dado, en el sentido de que no todos los integrantes de la red interactúan al mismo nivel. Y si se asume a la red de política como una estructura dinámica, se podrían observar las cualidades estructurales de la red a lo largo de ciertos periodos y determinar la variabilidad (o no) en los modos de interacción. 


\section{Bibliografía}

Aguilar, V.L.(2014). «Las dimensiones y los niveles de la gobernanza», en Cuadernos de Gobierno y Administración Pública, Vol. 1, No. 1.

Allison, G. T. (1969). «Conceptuals models and the cuban missils crisis», en The American Political Science Review, vol. LIII, n 3.

Aragón, D.A. (2001). «Estructuras de gestión en redes interorganizacionales: el caso del programa de coinversión social del gobierno del DF/NOVIB y organizaciones civiles», Tesina (Maestría en Administración y Políticas Públicas). CIDE, México.

Bardach, E. (1978). The implementation game. MIT Press, Cambridge.

Berardo, R. (2009). «Sustaining joint ventures: the role of resource exchange and the strength of inter-organizational relationships», en Feiock, R. y J.T. Scholz (eds.). Self-organizing federalism: collaborative mechanisms to mitigate institutional collective action. Cambridge University Press, Cambridge.

Bravo, J. (1997). «Redes inter-organizacionales y redes de políticas. Una revisión inicial», Documento de Trabajo, No. 53. CIDE, México.

Burt, R.S. (1997). «The contingent value of social capital», en Administrative Science Quarterly, No. 42.

Burt, R.S. (2000). «The network structure of social capital», en Research in Organizational Behavior, 22.

Carlsson, L. (2000). «Policy networks as collective action», en Policy Studies Journal, vol. 28. $n^{0} 3$.

Coleman, J. (1988). «Social Capital in the Creation of Human Capital», en American Journal of Sociology, n ${ }^{\circ} 94$.

Coleman, J.S. (1990). Foundations of social theory. Harvard University, Cambridge.

Crozier, M. Y E. Friedberg (1980). The actor and the system. University of Chicago Press, Chicago.

Del Castillo, A. (2000), «Problemas en la acción gubernamental: organizaciones y redes de actores», en Arellano, D.; E. Cabrero y A. Del Castillo (coords.). Reformando al gobierno. Una visión organizacional del cambio gubernamental. CIDE/Porrúa, México.

Evans, M. (1998). «Análisis de redes de políticas públicas: una perspectiva británica», en Gestión y Política Pública, vol. VII, $\mathrm{n}^{\circ} 2$.

García, O.M. (2009). «Visualización del conocimiento sociológico. Análisis de redes sociales». URL: http://visualizacionsociologia.blogspot.com, última consulta 10 de Mayo de 2010.

Granovetter, M. (1973). «The strength of peak ties», American Journal Sociology, nº 78.

Guarneros, M.V. (2007). «Urban governance and participation in central Mexico», en Development, $\mathrm{n}^{\circ} 50$, vol. 1 .

Hanneman, R.A. y Mark R. (2005). Introduction to social network methods. University of California, CA.

Hardin, G. (1968). «The tragedy of the commons», en Science, $\mathrm{n}^{\circ} 162$.

Howlett, M. (2002). «Do networks matter? Linking policy network structure to policy outcomes: evidence from four canadian policy sectors 1990-2000», en Canadian Journal of Political Science, $\mathrm{n}^{\mathrm{o}}$ 35, Vol. I.

Jones, C., W. Hesterly y S. Borgatti (1997). "A general theory of network governance: exchange conditions and social mechanisms», en Academy Of Management Review, ${ }^{\circ}{ }^{2} 2$, vol. 4.

Kickert, W.J.W., Klijn, E. H. y Koppenjan, J. F. M. (1997). «Managing networks in the public sector: findings and reflexions», en Kickert, W .J. M., Klijn, E. H. y Koppenjan, J. F. M. (eds.). Managing complex networks. Strategies for the public sector. SAGE Publications, Londres. 
Klijn, E. H. (1997). «Policy networks: An overview», en Kickert, W.J.M., Klijn, E. H. y Koppenjan, J. F. M. (eds.). Managing complex networks. Strategies for the public sector. SAGE Publications, Londres

Knoke, D. (1990). Political networks. The structural perspective. Cambridge University Press, Cambridge.

Knoke, D. et al. (1996). Comparing policy networks: Labor politics in the U.S., Germany, and Japan. Cambridge University Press, Cambridge.

Knoke, D. y Yang, S. (2008). Social network analysis. SAGE Publications, Los Angeles.

Lewis J.M. (2005). «A network approach for researching partnerships in health», en Australia and New Zealand Health Policy, $\mathrm{n}^{\circ}$ 22, vol. 2.

Lindblom, C. E. (1979). «Still muddling, not yet through» en Public Administration Review, vol. $39, \mathrm{n}^{\mathrm{o}} 6$.

Luna, M. y Velasco, J. L. (2009). «Las redes de acción pública como sistemas asociativos complejos: Problemas y mecanismos de integración», en Revista Hispana para el Análisis de Redes Sociales, vol. 17, $\mathrm{n}^{\circ} 4$.

Luna, M. y Velasco, J. L. (2009). «Las redes de acción pública como sistemas asociativos complejos: problemas y mecanismos de integración», en Revista Hispana para el Análisis de Redes Sociales, $\mathrm{n}^{\mathrm{0}} 17$, vol. 4.

Luna, M. (2005). «Las redes de acción pública: ¿un nuevo ‘circuito’ de la ciudadanía?, en Arditi, B. (ed.). ¿Democracia post-liberal? El espacio político de las asociaciones. Editorial Anthropos/ Fac. Ciencias Políticas y Sociales-UNAM, México.

March, J.G. y Olsen, J. P. (1989). Rediscovering institutions: the organizational basis of politics. The Free Press, New York.

Mariñez, F. (2007). «Capital social y redes de políticas. Acciones públicas en la zona metropolitana de Monterrey, Nuevo León», en Región y Sociedad, vol. XIX, no 39.

Mayntz, R. (2000). «Nuevos desafíos de la teoría de governance», en Instituciones y Desarrollo, $\mathrm{n}^{\mathrm{o}} 7$.

Méndez, J.L. (2000). «La política como variable dependiente: hacia un análisis más integral de las políticas públicas», en J.L. Méndez (comp.). Lecturas básicas de administración y políticas públicas. El Colegio de México, México.

Mintzberg, H. (1979). The structuring of organizations. Prentice-Hall, New Jersey.

Mouffe, C. (2000). La paradoja democrática. Gedisa, España.

Natal, A. (2001). «How state funds impact NGO's capacity to foster people's participation». Documento de Discusión sobre el Tercer Sector. No. 12. México: El Colegio Mexiquense.

Natal, A. (2007). Lecciones de la teoría de dependencia de recursos para el estudio de las OSC mexicanas. (mimeo).

Natera, P.A. (2005). «Nuevas estructuras y redes de gobernanza», en Revista Mexicana de Sociología, n ${ }^{\circ}$ 67, vol. 4.

Nigenda, G. y L.M. González, (2009), «Contracting private sector providers for public sector health services in Jalisco, Mexico: perspectives of system actors», en Human Resources for Health, $\mathrm{n}^{\mathrm{0}}$ 7, vol. 79.

Olson, M. (1965). The logic of collective action: Public goods and the theory of groups. Harvard University Press, Cambridge.

Ostrom, E. (1990). Governing the commons. Cambridge University Press, Cambridge.

Percival, G. (2009). «Exploring the influence of local policy networks on the implementation of drug policy reform: the case of California's substance abuse and crime prevention act», en Journal of Public Administration Research and Theory, $\mathrm{n}^{\circ}$ 19, vol. 4.

Pfeffer, J. y Salancik, G. R. (1978). The external control of organizations. A resource dependence perspective. Harper and Row, New York. 
Poggiese, H.; Redín, M. E. y P. Alí (1999). «El papel de las redes en el desarrollo local como prácticas asociadas entre Estado y sociedad», en www.flacso.org.ar/areasyproyectos/ (consultado por última vez el 08 de abril de 2010, formato .pdf).

Porras, F. (2007). «Rethinking local governance: hierarchies and networks in mexican cities», en European Review of Latin American and Caribbean Studies, $\mathrm{n}^{\circ} .83$.

Porras, M.J.I (2001), «Policy network o red de políticas públicas: una introducción a su metodología de investigación», en Estudios Sociológicos, vol. XIX, nº 57.

Pressman, J.L. y Wildavsky, A. (1973). Implementation: how great expectations in Washington are dashed in Oakland; or, why it's amazing that federal programs work at all. California University Press, Berkeley.

Provan, K.G. y Milward, H. B. (1995). «A preliminary theory of network effectiviness: A comparitive study of four community mental health systems», en Administrative Science Quaterly, no 40, vol. 1.

Putnam, R. (1993). «The prosperous community: social capital and public life», en The American Prospect, $\mathrm{n}^{\mathrm{o}} 13$.

Putnam, R. D. (1995). «Bowling alone: America's declining social capital», en Journal of Democracy, vol. 6, $\mathrm{n}^{\mathrm{B}} 1$.

Rhodes, R. (1997), Understanding governance. Policy networks, governance, reflexivity and accountability. Open University Press, Buckingham.

Sánchez, P.G. (2008), La implementación de programas públicos a través de redes de política en los municipios mexicanos: dos casos de estudio, Tesina (Maestría en Administración y Políticas Públicas). CIDE, México.

Sandström A. y Rova, C. (2010). «The network of adaptive governance: a single case study of a fish management area», en International Journal of the Commons, vol. $4, \mathrm{n}^{\circ} 1$.

Sandström, A. (2008). Policy networks: the relation between structure and performance. Suecia: Luleå University of Technology. Tesis Doctoral

Sandström, A. y Carlsson, L. (2008). «The performance of policy networks: the relation between network structure and Network performance», en The Policy Studies Journal, vol. $36, \mathrm{n}^{\mathrm{o}} 4$.

Scharpf, F. (1997). Games real actors play; actor-centered institutionalism in policy research. Westview Press, Boulder.

Simon, H.A. (1976). Administrative behavior: a study of decision making processes in administrative organization. Free Press, New York.

Stoker, G. (1998). «El buen gobierno como teoría: cinco propuestas», en Revista Internacional de Ciencias Sociales, $\mathrm{n}^{\circ} .155$.

Teisman, G. R y Klijn, E. H. (2002). «Partnership arregements: governmental rhetoric or governance scheme», en Public Administration Review, en vol. 62, $\mathrm{n}^{\mathrm{o}} 2$.

Teisman, G.R (2000). «Models for research into decision-making processes: On phases, streams and decision-making rounds», en Public Administration, vol. 78, $\mathrm{n}^{\circ} .4$.

Themudo, N. (2000). NGO and resources: Getting a closer grip on a complex are. Documento de Discusión sobre el Tercer Sector. No. 5. México: El Colegio Mexiquense.

Vargas, S. (2008). «Redes de políticas y cambio organizacional en la política forestal mexicana», en Gestión y Política Pública, n ${ }^{\circ}$ VII, vol. 1.

Vázquez, A. (2010). La dependencia entre organizaciones de la sociedad civil en procesos de participación ciudadana. Caso pueblos mágicos en Valle de Bravo, 2004-2006. Universidad Autónoma del Estado de México, México.

Wasserman, S. y Faust, K. (1994). Social network analysis: methods and applications. Cambridge University Press, Cambridge. 
Zabaleta, D. (2008). La gestión de redes como una estrategia gubernamental de liderazgo contingente: dos proyectos de conservación del patrimonio cultural urbano en la Ciudad de México, Tesina (Maestría en Administración y Políticas Públicas). CIDE, México.

Zabaleta, S.D. (2009). «Algunas implicaciones normativas de las redes de política desde la noción de espacio público», en Pardo, M. del C. (coord.). Nuevas tendencias en la teoría y en la práctica de la administración y de las políticas públicas. El Colegio de México, México.

Zurbriggen, C. (2003). Las redes de políticas. Una revisión teórica, Documento de Trabajo, (s.n.), en http://guajiros.udea.edu.co/fnsp/cvsp/politicaspublicas (consultado por última vez el 12 de noviembre de 2009, formato .pdf). 